\title{
Studies on multi-scale full waveform inversion for time-domain acoustic wave equation based on virtual-source precondition
}

\author{
Mengxue Dai, Jingbo Chen \\ Key Laboratory of Petroleum Resources Research \\ Institute of Geology and Geophysics, Chinese Academy of Sciences \\ Beijing, China \\ daimengxue@mail.iggcas.ac.cn
}

\begin{abstract}
Time-domain multi-scale full waveform inversion (FWI) is an important method for seismic imaging. Using different frequency contents of seismic data, this method mitigates the effect of the local-minima problem by sequential inversion. One of the important factors determining the convergence rate of the nonlinear inversion is the precondition of the gradient. Virtual-source precondition has been successfully applied to Laplace-domain and Laplace-Fourier-domain FWI. In this paper, we will examine the application of the virtual-source precondition to time-domain multi-scale FWI. The research results reveal that, compared to the gradient method without precondition, the virtual-source-based gradient method achieves much greater convergence rate while its additional computational cost is very low. Therefore, virtual-source precondition is very effective for time-domain multi-scale FWI.
\end{abstract}

keywords-virtual-source; precondition; full-waveform inversion

\section{INTRODUCTION}

Seismic waveform contains a lot of information which is ignored in conventional inversion. Full waveform inversion (FWI) is a data-fitting method based on full wavefield modeling to obtain quantitative information of the underground structure ${ }^{[1]}$.

Frequency domain FWI was proposed by Pratt ${ }^{[2]}$, it uses implicit finite-difference scheme and is convenient in multishots computing and attenuation, but occupies large memory in wavefield modeling. Time domain FWI was proposed by Tarantola ${ }^{[3]}$, it uses explicit finite-difference scheme, occupies less memory in wavefield modeling and is good for computing large $3 \mathrm{D}$ problems. We proceed FWI in time domain in this paper.

In the inversion of complex model, the misfit function has many local minimums, thus the inversion may easily stuck in local minimum instead of converging to global minimum. To solve this problem, Bunks ${ }^{[4]}$ proposed multi-scale FWI method. This method abstracts different frequency components from seismic data by low-pass filtering, then proceeds sequential inversion from low frequency to high frequency. As a result, the local minimum problem in non-linear inversion is mitigated, the dependence on initial model is reduced, and FWI method can converge to global minimum stably. Boonyasiriwat ${ }^{[5]}$ did some further research on the low-pass filtering method and frequency band chosen strategy.

The precondition of the gradient is one of the important factors determining the convergence rate of the nonlinear inversion. The precondition factor can remove the effect of geometric spreading from source to the deep part of model, rebalance the deep and shallow scatterers' contribution to gradient, and make the inversion converge faster ${ }^{[6]}$. Shin ${ }^{[7]}$ proposed virtual-source precondition, which is constituted by the reverse of the diagonal of the pseudo-Hessian matrix. Shin and Cha ${ }^{[8]}{ }^{[9]}$ applied virtual-source precondition in Laplace domain and Laplace-Fourier domain FWI, and achieved ideal result. In this paper, we applied the virtual-source precondition in time domain FWI, and compared the results between time domain FWI with virtual-source precondition and time domain FWI without precondition through the inversion of Marmousi model.

\section{Time DOMAin Multi-Scale FWI}

In this paper, we use the time domain multi-scale FWI method in Bunks' paper ${ }^{[4]}$, but in the forward modeling part, we use 8-th order finite-difference scheme instead of the original 2-th order finite-difference scheme.

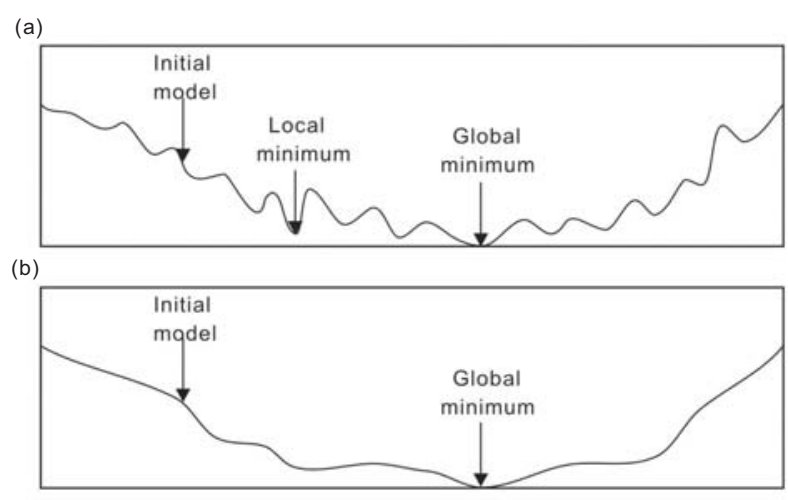

Fig. 1. Illustration of multi-scale method (a) higher frequency; (b) lower frequency. 
The time domain acoustic equation is

$$
\frac{1}{v^{2}} \frac{\partial^{2} p}{\partial t^{2}}=\left(\frac{\partial^{2} p}{\partial x^{2}}+\frac{\partial^{2} p}{\partial z^{2}}+s\right)
$$

where $p$ is the wave-field, $s$ is the source, $t$ is the time, $v$ is the velocity, $X$ is the horizontal distance, $Z$ is the vertical distance.

As in Bunks' paper ${ }^{[4]}$, the expression of gradient is

$$
\operatorname{grad}=\frac{2}{v^{3}} \int_{0}^{T} \lambda \frac{\partial^{2} p}{\partial t^{2}} d t
$$

where ${ }^{p}$ can be obtained by solving (1), $\lambda$ can be obtained by solving the adjoint equation

$$
\frac{1}{v^{2}} \frac{\partial^{2} \lambda}{\partial t^{2}}=\left(\frac{\partial^{2} \lambda}{\partial x^{2}}+\frac{\partial^{2} \lambda}{\partial z^{2}}\right)+(p-\tilde{p})
$$

where $\tilde{p}$ and $p$ are the observed and modeled data.

As illustrated in Fig. 1a, the higher the frequency used in inversion, the more local minimums in misfit function, so the inversion may easily stuck in the local minimum. As illustrated in Fig. 1b, the lower the frequency used in inversion, the more smooth the misfit function will be, so the inversion can converge to the global minimum in stable manner. Thus, we use the multi-scale method step by step: first we carried out the inversion in $\mathrm{n}$ the longest scale and obtain a model close to the global minimum, then use this model as the initial model and proceed inversion in shorter scales. This method reduces the dependence of initial model, can converge to global minimum stably and obtain a satisfying result.

\section{VIRTUAL-SOURCE PRECONDITION}

In the situation of classical acoustic or elastic wave, the contribution to the gradient of deep scatterers is usually less than shallow scatterers. Because the amplitude of the wave diminishes when the wave spread into the distance. The precondition factor can remove this effect of geometric spreading to make the inversion converge faster.

The inverse of the diagonal of the Hessian matrix is often used as the precondition of gradient, but the computation is complex and expensive. Shin[7] proposed the virtual-source precondition in prestack depth migration. The virtual-source precondition uses the diagonal of the pseudo-Hessian matrix, it is concise and easy to compute.

In Laplace domain, the expression of virtual-source is ${ }^{[8]}$

$$
\mathbf{f}_{x, z}=-\frac{\partial \mathbf{Z}}{\partial v_{x, z}} \mathbf{p}
$$

where $\mathbf{f}_{x, z}$ is the virtual source at location $(x, z), z$ is the impedance matrix, $v_{x, z}$ is the velocity at location $(\mathrm{x}, \mathrm{z}), \mathbf{p}$ is the modeled wavefiled. The precondition factor constructed by virtual source is

$$
\sum_{i=1}^{n_{s}}\left(\mathbf{f}_{x, z}\right)^{T} \mathbf{f}_{x, z}+\beta
$$

where $\beta$ is used to make sure the items of the precondition matrix are not zero. $\sum_{i=1}^{n_{s}}$ denotes the sum of shots.

In time domain, we obtain the expression of virtual-source by taking the partial derivative of (1) with respect to the velocity:

$$
\mathbf{f}_{x, z}=\frac{2}{v^{3}} \frac{\partial^{2} p}{\partial t^{2}}
$$

Thus, the precondition factor is

$$
\sum_{i=1}^{n_{s}}\left(\mathbf{f}_{x, z}\right)^{T} \mathbf{f}_{x, z}+\beta=\sum_{i=1}^{n_{s}}\left(\frac{2}{v_{x, z}{ }^{3}} \frac{\partial^{2} p_{x, z}}{\partial t^{2}}\right)^{2}+\beta
$$

The time domain finite-difference gradient with virtualsource precondition is

$$
\operatorname{grad}=\frac{\sum_{i=1}^{n_{s}} \sum_{t=0}^{T} \lambda_{x, z}^{t} \frac{2}{v_{x, z}^{3}}\left[\frac{p_{x, z}^{t+1}-2 p_{x, z}^{t}+p_{x, z}^{t-1}}{\Delta t^{2}}\right]}{\sum_{i=1}^{n_{s}} \sum_{t=0}^{T}\left(\frac{2}{v_{x, z}^{3}}\left[\frac{p_{x, z}^{t+1}-2 p_{x, z}^{t}+p_{x, z}^{t-1}}{\Delta t^{2}}\right]\right)^{2}+\beta}
$$

\section{EXAMPLES}

We use the regenerated Marmousi model, the grid size is $n z \times n x=94 \times 175$, the grid intervals are $\Delta x=\Delta z=6 m$, as illustrated in Fig. 2.

Set a receiver at each grid point at $n z=1$, added up to 175 receivers. Set 25 shots at $n z=2$, the interval between shots is $30 \mathrm{~m}$. The source is a Ricker wavelet with a central frequency of $30 \mathrm{~Hz}$. The sampling time interval is $0.0004 \mathrm{~s}$, the recording time is $0.56 \mathrm{~s}$. As shown in Fig. 3, the initial model is a laterally homogeneous model with velocity linearly increasing with depth. The velocity at the surface and the bottom is $1500 \mathrm{~m} / \mathrm{s}$ and $4300 \mathrm{~m} / \mathrm{s}$, respectively.

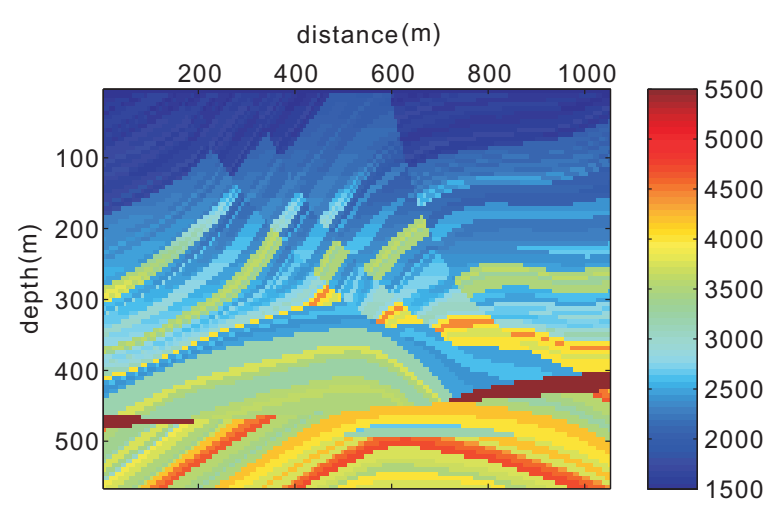

Fig. 2 The regenerated Marmousi velocity model 


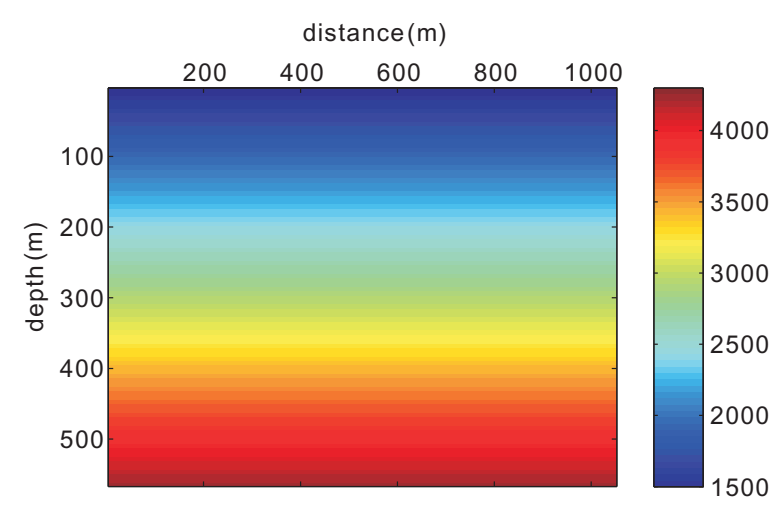

Fig. 3 Initial linearly increasing velocity model

We use multi-scale inversion method. First, we abstract 0$10 \mathrm{~Hz}, 0-15 \mathrm{~Hz}$ frequency component from the source wavelet with a central frequency of $30 \mathrm{~Hz}$ by low-pass filtering. Then, we proceed inversion in different scales using source wavelet filtered from $0-10 \mathrm{~Hz}, 0-15 \mathrm{~Hz}$, and unfiltered wavelet in sequence. The iteration number of different scales is 40,40 , and 60 , respectively.
We conducted the inversion process mentioned above using multi-scale FWI no-precondition and virtual-source preconditioned gradient method. As illustrated in Fig. 4, the three left pictures are the results using no-precondition gradient method after 40 iterations in $0-10 \mathrm{~Hz}, 40$ iterations in $0-15 \mathrm{~Hz}$, and 60 iterations in $30 \mathrm{~Hz}$ central frequency, respectively. The three right pictures are the results using virtual-source preconditioned gradient method after 40 iterations in $0-10 \mathrm{~Hz}$, 40 iterations in $0-15 \mathrm{~Hz}$, and 60 iterations in $30 \mathrm{~Hz}$ central frequency, respectively. We can see from the pictures that the result using virtual-source preconditioned gradient method is more accurate and closer to the true model. Especially for the deep part of the model, the result using no-precondition gradient method can only present a coarse tendency and the velocity is not right. However, in the result using virtual-source preconditioned gradient method, the anticlines in the deep part are well imaged.

Fig. 5 illustrates the two methods' convergence curves of the three scales. The green line represents no-precondition gradient method, the red line represents the virtual-source preconditioned gradient method. As shown in the figure, virtual-source preconditioned gradient method converges faster and more stable than no-precondition gradient method.

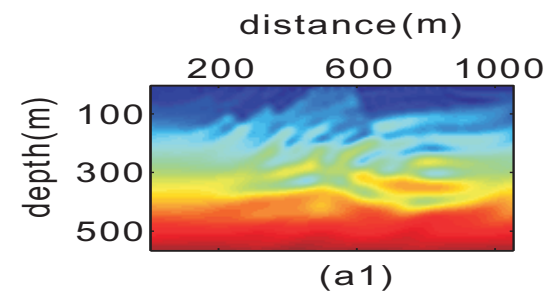

4000
3000
2000

(a1)

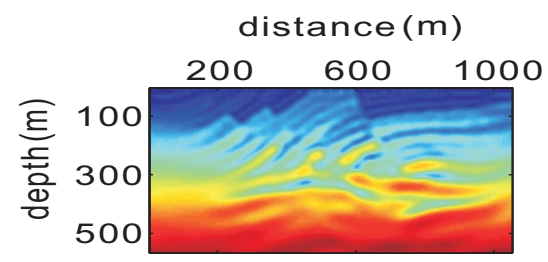

(b1)

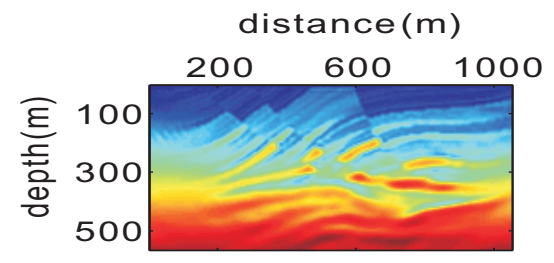

(c1)

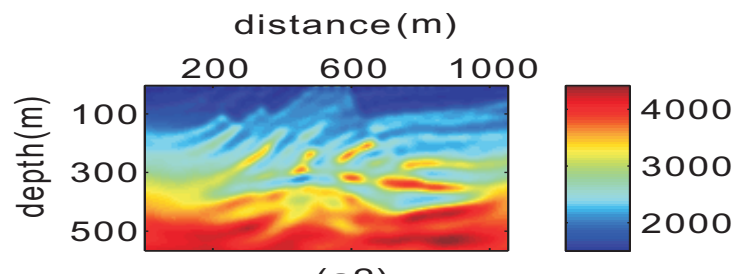

(a2)

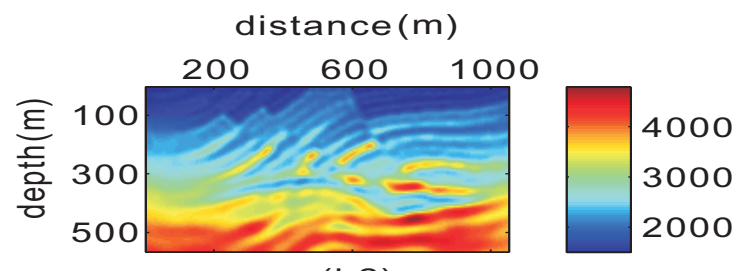

(b2)

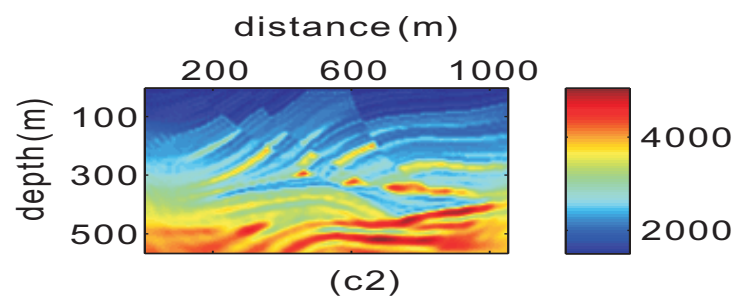

Fig. 4 Comparison between results of without and with virtual-source precondition gradient multi-scale FWI method (a1) velocity model after 40 iterations, gradient method without precondition, $0-10 \mathrm{~Hz}$ data (b1) velocity model after 40 iterations, gradient method without precondition, 0-15Hz data (c1) velocity model after 60 iterations, gradient method without precondition, unfiltered data (a2) velocity model after 40 iterations ,gradient method with virtual-source precondition, $0-10 \mathrm{~Hz}$ data (b2) velocity model after 40 iterations, gradient method with virtual-source precondition ,0-15Hz data (c2) velocity model after 60 iterations ,gradient method with virtual-source precondition ,unfiltered data 

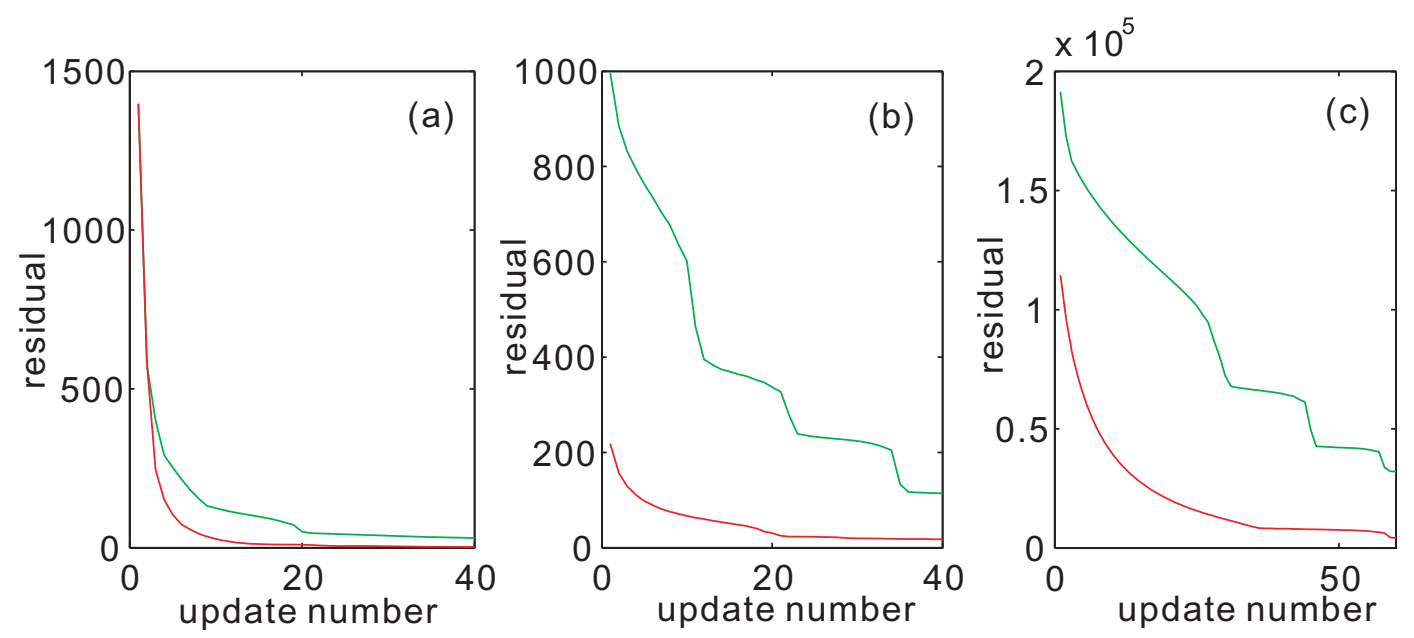

Fig. 5 Comparison of convergence curves (a) $0-10 \mathrm{~Hz}$ (b) $0-15 \mathrm{~Hz}$ (c) unfiltered data

To complete one inversion iteration, the average time noprecondition gradient method needs is 659s, the average time virtual-source preconditioned gradient method needs is $765 \mathrm{~s}$, the ratio is about $8.6: 10$, the additional computational cost increase is very low.

\section{CONCLUSIONS}

In this paper, we applied the virtual-source precondition in time domain multi-scale FWI, and compared virtual-source preconditioned gradient method with no-precondition gradient method. The result shows that with virtual-source precondition, the multi-scale FWI converges faster and more stable than the inversion without precondition, the additional computational cost increase is very low, and the inversion result approves a lot.

\section{ACKNOWLEDGMENT}

We would like to thank the reviewers for helping us to improve the paper.

\section{REFERENCES}

[1] J. Virieux and S. Operto, "An overview of full-waveform inversion in exploration geophysics,” Geophysics, 74(6):WCC1-WCC26, 2009.

[2] R. G. Pratt and M. H. Wothington, "Inverse theory applied to multisource cross-hole tomography, Part 1: Acoustic wave equation method,” Geophysical Prospecting, 38(3): 287-310, 1990.

[3] A. Tarantola, "Inversion of seismic reflection data in the acoustic approximation,” Geophysics, 49(8): 1259-1266, 1984.

[4] C. Bunks, F. M. Saleck, S. Zaleski, and G. Chavent, "Multiscale seismic waveform inversion," Geophysics, 60(5):1457-1473, 1995.

[5] C. Boonyasiriwat, P. Valasek, P. Routh, W. Cao, G. T. Schuster, and B. Macy, "An efficient multiscale method for time-domain waveform tomography,” Geophysics, 74(6):WCC59-WCC68, 2009.

[6] E. Causse, R. Mitte, and B. Ursin, "Preconditioning of full-waveform inversion in viscoacoustic media,” Geophysics, 64(1): 130-145, 1999.

[7] C. Shin, S. Jang, and D. J. Min, "Improved amplitude preservation for prestack depth migration by inverse scattering theory," Geophys Prospecting, 49:592-606, 2001.

[8] C. Shin and Y. H. Cha, "Waveform inversion in the Laplace domain," Geophys. J. Int., 173(3): 922-931, 2008.

[9] C. Shin and Y. H. Cha, "Waveform inversion in the Laplace-Fourier domain,” Geophys. J. Int., 177(3):1067-1079, 2009. 